\title{
A Research on Organizational Evolution and Upgrading Strategies of Flower Industrial Cluster
}

\author{
Wei Jianfeng* \\ College of Business Administration, Henan University, Kaifeng, Henan, 475004, China
}

\begin{abstract}
Through investigation on farmer, company and relevant bureau of local government, an analysis was conducted on organizational evolution of flower industrial cluster through induction and statistical methods. The research indicated that the organizational scale and structure had been unceasingly evolved during the process resulting in such industrial cluster. In recent years, the industrial cluster has been challenged for its sustainable development, which then has slowed down due to the decreasing business income. The challenge not only resulted from declined growth in market demand, rising factor price and other external environmental factors, but also is relevant to organizational structure of cluster, quality of product, innovative capability and other internal factors. Therefore, in order to maintain sustainable competitive advantages, the flower industrial cluster needs to implement innovation incentive strategy and update organizational structure, production mode and quality of product.
\end{abstract}

Keywords: Flower industrial cluster, organizational evolution, problem and challenge, upgrade strategy.

\section{INTRODUCTION}

In developed countries, agricultural industrial cluster is a common phenomenon. Since 1980s, Chinese agricultural industrial cluster has gradually formed and rapidly developed. These clusters primarily exist in featured agricultural field, such as Shouguang vegetable industrial cluster in Shandong, Lingbao apple industrial cluster in Henan, Dounan Flower industrial cluster in Yunan, etc. Since $21^{\text {st }}$ century, more and more foreign and domestic scholars have turned their attention to agricultural industrial cluster. Their researches have been mainly focused on formation mechanism and competitive advantage of cluster. For example, Lee and Schrock (2002) built cluster model to explain the effect of historic knowledge of production, technology and market on formation of rural industrial cluster [1]. As the rural industrial cluster has been constantly evolving, its growth mechanism and innovative impetus has attracted the attention of many scholars. For example, Zheng Fengtian and Cheng Yu took the generation and growth of flower industrial cluster in Dounan, Yunan province as research case to dissect the effect of entrepreneur during generation and evolution of such cluster [2]. Li Erling el took the flower industrial cluster in Yanling county, Henan province as an example to analyze agricultural innovative architecture of agricultural industrial cluster [3]. Recently, there are some deficiencies as follows: (1) few literatures about analysis on evolution and reason of agricultural industrial cluster structure; (2) lack of research on impartible relation between externally environmental change and

*Address correspondence to this author at the College of Business Administration, Henan University, Kaifeng, Henan, 475004, China; Tel/Fax: 86-15093677379; E-mail: weijianfenghd@sina.com internal restriction factors; (3) lack of pertinence in research of upgrade strategy of agricultural industrial cluster.

This article intended to take Yanling flower industrial cluster in China as an example and structural evolution as its principal line to conduct analysis on evolution, external environmental change and internal restriction factors of agricultural industrial cluster, and to put forward the upgrade strategies based on the previous results. This research study aimed to thoroughly understand the developmental context, problems and challenges regarding Chinese agricultural industrial cluster, supplement the inadequacy of relevant theoretical research and provide basis for local government to formulate relevant promotion policy and enterprise strategic decision.

This article adopted the following research methods: on one hand, in-depth investigations were conducted on personnel concerned from local government and agricultural competent department, and on cluster enterprise operator and farmer of Yanling flower industrial cluster in order to acquire first-hand information; on the other hand, several inquiries and researches were carried on relevant literature as well as documents from Yanling agricultural competent department, websites on flower and flower hi-tech park in order to obtain relevant data; finally, on these bases, a theoretical analysis was made with a conclusion based on statistical and deductive methods.

\section{STRUCTURAL EVOLUTION OF FLOWER INDUSTRIAL CLUSTER}

\subsection{Structural Scale}

Since initial restorative development stage (1978-1985), Yanling flower industry experienced primary development 
stage (1985-1995), introductory development stage (19961998), rapid development stage (1999-2006) and stable development stage (2007-2011). However after 2012, this scale was basically stabilized. In 1999, the flower planting area was $4600 \mathrm{hm} 2$, and then it boomed quickly and reached to $25467 \mathrm{hm} 2$ till 2004. By the end of 2009, in Yanling, the flower planting area had expanded from traditional 4 to 12 villages and towns; specialized flower villages had been extended to 122; there had been various flower companies, gardens, farms and nurseries amounting to 7837, which owned a total planting area of $38667 \mathrm{hm}^{2}$ in which more than 2300 plant varieties were planted including ornamental nursery stock, lawn, bonsai and fresh cut flower series and 1.8 billion plants (pot) were produced annually [4]. In 2011, the planting scale of Yanling flower industrial cluster had expanded to $41227 \mathrm{hm}^{2}$, which occupied $37.7 \%$ of flower area in Henan province and $5.5 \%$ of Chinese total flower area; the output value of flower occupied $51.2 \%$ of total agricultural output and the number of employees reached 200,000. Table 1 represents the planting area, output value and proportion of agricultural output value of Yanling flower industrial cluster over the years.

The main factors of the formation and rapidly expanded scale of Yanling flower industrial cluster include: (1) Demand pull: after 1990s, with accelerated urbanization and improved living standards of people in China, the flower market scale expanded rapidly, creating a good external environment for the development of flower industry. (2) Physiographic condition: Yanling is a warm temperate monsoon climate and a Chinese typical transition zone from southern climate type to northern climate type, which forms a natural domestication base to transplant Southern Flower in northern zone or Northern Flower in southern zone. After domestication and cultivation, the nursery stocks in Yanling obtain a high rate of survival in Chinese Three North region as well as a vast market. (3) Planting tradition: the flower cultivation in Yanling can be dated back to Tang dynasty which prevailed in Song dynasty. Since ancient times, Yanling has been honored as the Capital of Flower or County of Flower, and it has acquired great fame for its Best Chimonanthus Praecox in China. The historic tradition and culture of flower planting in Yanling together with accumulated cultivation techniques have laid solid foundation for the formation of its flower industrial cluster. (4) Interest incentives: according to investigation by Yanling county government in 2008, the farmers' net income was 48,000 Yuan per hectare to plant flowers; the net income was over 15,000 Yuan per hectare to plant food crops like wheat and corn. Obviously, in that year, the net income to plant flowers was more than 3 times than that of common food crops. So, comparative income is an internal agent which enhances the scale of flower planting to expand rapidly. (5) Effect of cluster: the agglomeration effect produced by flower industrial cluster itself drove the scale of cluster to further expand. (6) Function of government: the Yanling county government promoted forward development of local flower industrial cluster through encouraging village cadres to take the lead to plant flowers and trees, implementing preferential policies to deduct or exempt land rent, establishing specialized flower trading market and flower technological park and other measures.

\subsection{Horizontal Structure of Organization}

With expanded scale of cluster, the internal structure (horizontal) of cluster has also gradually evolved. The initial formation of Yanling flower industrial cluster was impelled by farmers. Before 1995, only few farmers planted flowers and trees. Afterwards, farmers in adjacent areas imitated and

Table 1. Evolution of scale yanling flower industrial cluster.

\begin{tabular}{|c|c|c|c|}
\hline Year & Planting Area $\left(\mathbf{h m}^{2}\right)$ & Output Value (Ten Thousand YUAN) & $\begin{array}{c}\text { Proportion of Total Agricultural } \\
\text { Output Value (\%) }\end{array}$ \\
\hline \hline 1984 & 167 & 1000 & 4 \\
\hline 1995 & 1407 & 8000 & 6 \\
\hline 1998 & 3600 & 34000 & 15 \\
\hline 1999 & 4600 & 50000 & 35 \\
\hline 2004 & 25467 & 136000 & 35 \\
\hline 2005 & 26670 & 160000 & 38 \\
\hline 2006 & 30000 & 186000 & 38.3 \\
\hline 2007 & 34000 & 190000 & 39 \\
\hline 2008 & 34933 & 247000 & 42.4 \\
\hline 2009 & 38667 & 300000 & 43.2 \\
\hline 2010 & 39333 & 302000 & 51.2 \\
\hline 2011 & 41127 & 370000 & \\
\hline
\end{tabular}


followed up the planting activity. Thus, a kind of small-scale planting pattern formed among farmers, which can be traced to the same origin with basic agricultural organizational form under Chinese household contract responsibility system. However, the organizational form of flower industry changed more rapidly than general planting industry. In the course of development of Yanling flower industrial cluster for over 20 years, its organizational form of flower production experienced an evolution process from traditional farmer to Farmer+Farmer (cooperation of famers), Farmer+Agricultural Enterprise, Cooperative+ Agricultural Enterprise, and Modern Agricultural Enterprise [5]. Recently, the internal structure of cluster is a pattern of symbiosis of famers, agricultural enterprises and various cooperative organizations. In which, the cooperative organization includes two types: jointly operated organization composed under input of land and original capital, and enterprise association or alliance composed by enterprises and farmers through which members could share market information, technologies and orders, etc. Evolution of structure of cluster is presented in Fig. (1).

With expanded scale, there were huge changes in structure of Yanling flower industrial cluster. The impetus or basic approach of such change relies on: (1) the farmers transforming to enterprise by establishing companies after expanding their capital, technology and scale to achieve transformation from specialized household to enterprise. (2) The government attracting local enterprises to enter the cluster through construction of park zone so as to motivate emprise to rapidly expand their scale and effectively execute transformation from small nursery to modern agricultural enterprise. (3) The external scaled enterprise entering into this cluster. Such kind of enterprise may arise from the same industry out of this cluster or even come across different industries into this cluster. The external scaled enterprises entered and brought new knowledge, techniques and management and operation mode, and made prominent contributions on scale, and normalized and facilitated agricultural construction. For example, in 2008, Henan Longyuan Flower Company settled in Yanling and built flower base of $3800 \mathrm{mu}$, which includes drip irrigation of $500 \mathrm{mu}$ and spray irrigation of $3000 \mathrm{mu}$; its Chinese leading facility construction and normalized planting mode provided guidance and demonstration effect for the development of cluster.

\subsection{Vertical Structure of Organization}

The evolution of vertical structure of cluster is primarily manifested as an extended industrial chain, deepened vertical division of work and gradually improved support system. Yanling flower industry started with planting, and then further flourished with the formation of flower industrial cluster, which is shown in three aspects: (1) Gradually stretched industrial chain: with regard to seed breeding, a batch of breeding base arose, which indicated an extension from pure planting to hi-tech breeding. Simultaneously, the industrial chain also extended downstream into landscape engineering link having high profit and entry requirements. In recent years, landscape engineering enterprise has gradually increased and their qualification level has improved unceasingly. Besides, the deep processing industry of flower products also has improved to some extent, such as refining rose oil from roses. (2) Supporting organization and company grew out of nothing and developed step by step to provide support for development of industrial cluster. Within the cluster, a large number of agents are still the subjects to communicate with seller and buyer in trading, however, some professional flower dealers and logistic companies have already sprouted; the flower trading market coconstructed by local government and flower enterprises has gradually become a place for merchants to gather as well as an important trading place. A lot of dealers for the means of production of flower have settled in the cluster and provided services to planting enterprise. (3) The flower industry has driven development of hotel, catering and leisure and tourist industry. The Zhongyuan Flower Expo Park has been named as National Agricultural Tourism Model and National 4A Scenic Spot, and Huadu hot-spring resort has become the largest outdoor garden-like hot-spring resort in central plains of China. The extended industrial chain and gradually improved support system indicate a deepened and gradually matured agricultural industrial cluster. Fig. (2) illustrates the industrial chain and support system of flower industrial cluster.

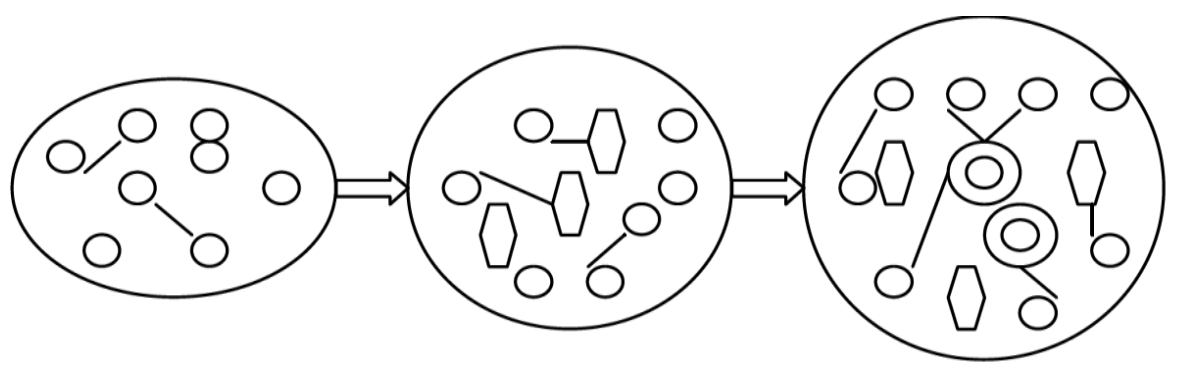

Legend: $\bigcirc$ Farmer $\square$ Medium $\quad$ ( Large Enterprise

\&Small Enterprise

Fig. (1). Evolution of structure of flower industrial cluster. 


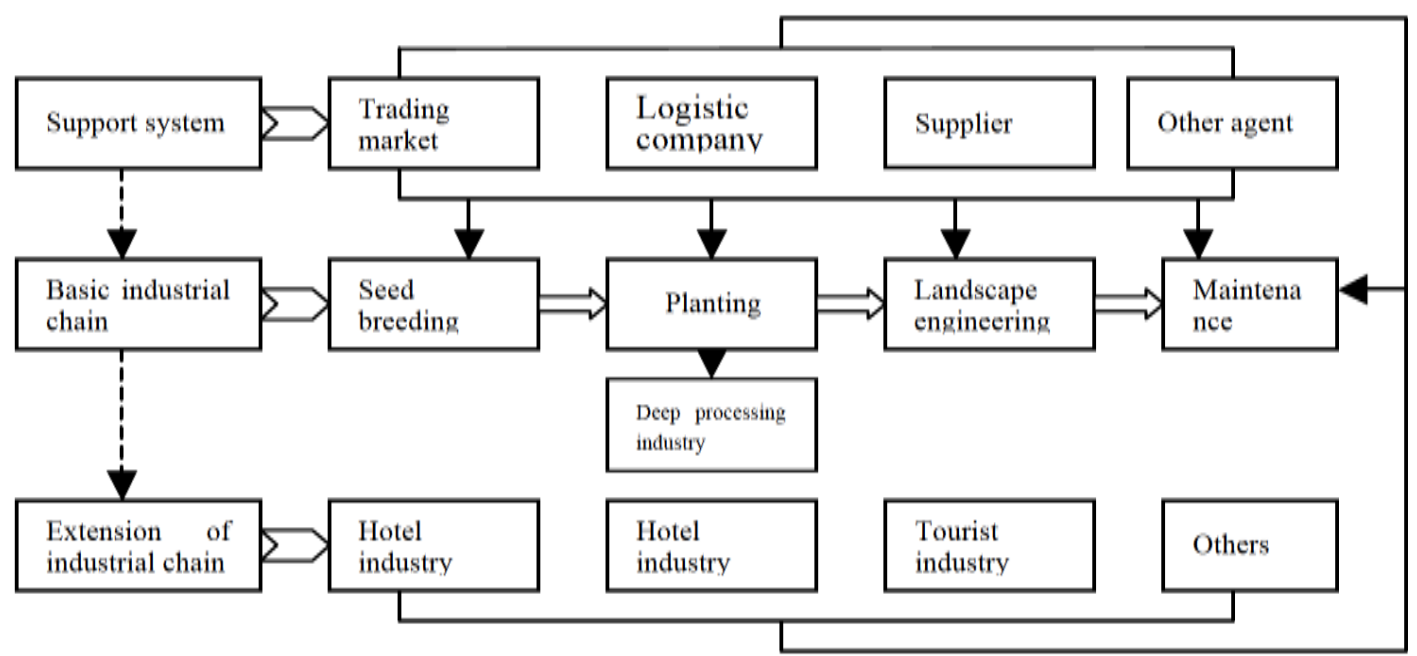

Fig. (2). Industrial chain and support system of flower cluster.

\section{PROBLEMS \& CHALLENGE}

\subsection{Market Needs and Production Factors}

\subsubsection{Changes of Condition of Needs}

Since 1990s, the quickened urbanization pace has improved living standards of the people and ecological construction need in China has propelled flower market to rapidly enlarge. Under this urging pressure, Chinese flower planting has rapidly expanded. After 2010, its supply and demand has gradually kept a balance, and recently it shows a situation of excessive supply, which is primarily presented by: (1) excessively increased supply; due to the higher income from nursery planting than that from planting crops, the flower industry still continuously attracts more farmers to enter into the enterprises, and every second or third industry of agricultural field also intends to enter into the flower planting industry. (2) Nursery market gradually transfers to buyer's market, which makes profit rate of partial nursery planting to decrease and even shows deficit. The competition of supplier is becoming increasingly intense. (3) Sophisticated and criticized needs, seasonality of flower products and structural surplus have emerged. As urban public landscape has become stable and real estate market has slowed down its growth speed, consequently, the nursery market has also retarded the growth. As for the price, large nursery is basically stable, but prices of small nursery have declined.

\subsubsection{Changes in Condition of Factors}

There is a basic trend of sophisticated needs and intensified market competition in landscape market; at the same time, the land rent, labor cost and other factor prices are continuously rising and vice versa. In recent years, the prices of labor, land rent, chemical fertilizers and other production materials have risen very high. Taking Yanling flower cluster as an example, daily salary per labor force (of temporary employee), land rent per mu and price of compound fertilizer have increased from 2009 to 2014, as shown in Table 2.

Under condition of unchanged sales prices, the rising factor cost would reduce profit margin of flower enterprise and farmers and make competition more intense.

The imbalance between supply and demand of flower products is relatively complex. Considering the supply, large nursery is featured by long cycle and high sunk cost and small nursery having a short production cycle, resulting in multiplicity of the elasticity of supply of flower products. In profit rising stage, there would be more incomers entering; supply of small nursery would be flexible in short term, while supply of large nursery would be lagging and inflexible in short term. In income declining section, supply would be lacking flexibility; though the rising product cost and falling profit may restrain enterprise to come, requiring a long term for reducing the supply. Due to low flexibility in demand of flower products, the stable demand could not only

Table 2. Factor price and changes unit: yuan (RMB).

\begin{tabular}{|c|c|c|c|}
\hline Year & Labor cost/ $\mathbf{h m}^{\mathbf{2}}$ & Land rent/ $\mathbf{h m}^{\mathbf{2}}$ & Compound fertilizer/ton \\
\hline \hline Year of 2009 & 450 & 9000 & 1700 \\
\hline Year of 2014 & 825 & 19500 & 2900 \\
\hline Rising rate (\%) & 83.3 & 117 & 70.6 \\
\hline
\end{tabular}


restrain further increase of product price, but also result in some product prices to rapidly decrease or even become lower than cost under condition of successive supply. In a word, the successive supply would be a long-term trend. After buyer's market forms, product quality and variety selection would be key factors of success.

\subsection{Internal Constraints}

\subsubsection{Organizational Structure}

The organizational structure is the core and basis of the problems. The organizational structure of Yanling flower industrial cluster has greatly changed in recent years. But as a whole, the production unit of single household still occupies absolute proportion in the cluster, and small-scale production mode is still the basic pattern of flower industrial cluster. Organizational structure of Yanling flower industrial cluster is shown in Table 3 .

Landscaping qualified enterprise with $\mathrm{C}+$ leveled qualification belongs to medium and large enterprise.

To diversify risks, the small-scaled operating farmers always adopt variety planting mode, which is contradictory to large-scale, professionalized and normalized planting idea and could not guarantee quality of product. Due to scattered economic subjects, imitation strategy is often applied that easily generates Herd Effect, namely following the trend of planting, thus resulting in imbalance of supply and demand of certain varieties between years. Once certain varieties supply more than demand, the prices sharply fall, resulting in the planter to face no profitor even severe loss. Smallscaled planting farmers often produce so limited quantities of products per batch that it becomes difficult to satisfy scale demand of engineering market; and seller and buyer may take multiple middle levels to complete trade, increasing searching and trading costs and bringing down earnings of planter.

\subsubsection{Industrial Chain and Support System}

For Yanling flower industrial cluster, though its professionalized work division level has been promoted to some extent, Small and Comprehensive production mode commonly exists and support system is not perfect. The principal problems are: (1) the slowly developing industry of anther, flower fertilizer, instrument, toll processing and other input cannot satisfy special demand of flower industrial cluster, and transportation cost is high for external purchase of production materials, which restrains comprehensive benefits for further promotion; (2) the shortage of specialized companies for planting, excavation, cultivation, loading, hoeing, watering and disease and pests prevention and treatment limits production quality and efficiency to increase; (3) deficient specialized market function, outdated flower trading means, lagged development of information, logistic and intermediary service industries and high trading cost; (4) the deep processing of flower products is just in emerging phase and industrial chain is too short.

\subsubsection{Product Variety and Quality}

Product structure and quality are key factors for the success of any enterprise. So is it in flower industry. The primary problems to affect competitive power of Yanling flower industrial cluster are: (1) the selection of variety lacks future orientation, as some varieties are excessively planted to result in excessive supply and some other varieties are disjointed with market demand and bad sell; (2) The lagged construction of product quality and packaging standards in production links, extensive management, not normalized cultivation techniques and outdated plant quarantine measures result in insufficient output of high-quality nursery stock and which is difficult to adapt to sophisticated and hypercritical market demand trend; (3) Unreasonable factor configuration, low degree of machinery operation as well as rising cost of employment increase production cost of products and affect market competition power.

\subsubsection{Innovation Capability}

Innovation capability is a critical factor for cluster to obtain sustainable competitive advantage. Recently, the key factors for restraining innovations of Yanling flower industrial cluster include: the farmers and small-scaled enterprises obtain planting and cultivation techniques and knowledge from their own planting experience summary and imitation, but majority of farmers have never received systematic training on specialized techniques, lacking new techniques and knowledge and processing low degree of scientist production management; though some large-scale enterprise could play a role of Technical Goalkeeper to some extent, they themselves still have some problems, such as weak technological power, weak technical research and development power, especially shortage of high-end technical and management talents, which result in lagged adoption, development and application of new techniques in the cluster which are hard to promote management level. Education level of employees in Yanling flower industrial cluster is shown in Table 4.

\section{UPGRADE STRATEGIES}

According to current development situation and future market trend of Yanling flower industrial cluster as well as

Table 3. Organizational structure of yanling flower industrial cluster.

\begin{tabular}{|c|c|c|c|c|}
\hline Type of organization & Large enterprise & $\begin{array}{l}\text { Medium \& small } \\
\text { enterprise }\end{array}$ & $\begin{array}{l}\text { Specialized household \& } \\
\text { cooperatives }\end{array}$ & Landscape enterprise \\
\hline Qty & 100 & 790 & 11000 & 38 \\
\hline
\end{tabular}

Data source: sorted by author according to relevant documents. 
Table 5. Education level of employees in Yanling flower industrial cluster.

\begin{tabular}{|c|c|c|c|c|}
\hline Education level & Primary school and below & Junior high school & Senior high school & $\begin{array}{c}\text { Technical secondary } \\
\text { school and university }\end{array}$ \\
\hline \hline Percentage & $16.11 \%$ & $49.63 \%$ & $29.98 \%$ & $4.27 \%$ \\
\hline
\end{tabular}

Data source: investigations by Yanling Agricultural Bureau in 2010.

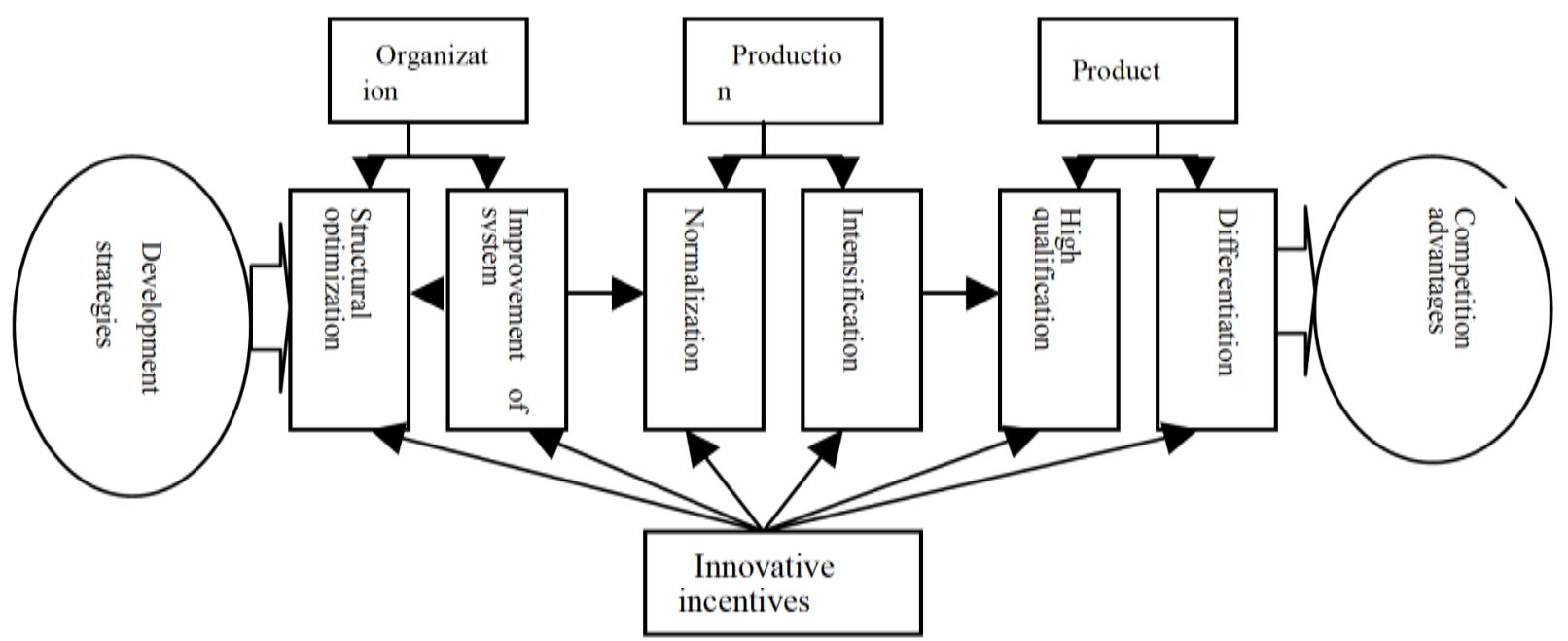

Fig. (3). Development strategies of flower industrial cluster.

basic evolution law of cluster, considering resistance against adverse effects after environmental factors, the author believes that this cluster requires to be strengthened in organizational structure, production mode and product and thus formulates its four major upgrade strategies, i.e. cluster organizational and structural optimization strategy, production normalization and intensification strategy, product structural optimization and quality promotion strategy and innovation driven strategy. Four strategies mutually promote and correlate as shown in Fig. (3).

\subsection{Structural Optimization and Improvement of System}

The innovation of cluster depends on conditions, process and relation structure stimulating the innovation activities [6], and the enhancement of innovation capability of cluster relies upon optimization of cluster structure and cultivation of organization [7]. One part of structural optimization is to push forward scale operation, which is the foundation of intensified production, normalized planting and technical innovation and also a vital condition to effectively improve product structure and reduce trading cost. An improved work division and support system is a competition advantage of cluster and also another strategic direction of structural optimization. The approaches to implement structural optimization include: (1) to attract external large-scale enterprise to enter into cluster through investment attraction is an important approach to accelerate cluster structural optimization; improving contract system, encouraging farmers and enterprises to enlarge current enterprise by holding share or becoming shareholder, and promoting farmer cooperative ventures to develop and expedite the course to transform farmers to enterprise; the transformation from farmer to enterprise is a basic direction of cluster evolution during which entrepreneurship may play an important role in such transformation course, so it is especially important to make efforts to create a local environment of entrepreneurship [7]. (2) The cluster production service industry plays an important role in reducing the production cost and trading expenses. Its key field is to develop cluster input industry, specialized production services company, specialized marketing company and logistic company in order to promote the building of electronic trading platform [8]. (3) The industrial chain extension and outward expansion could expand market space and promote competitive power and comprehensive benefits of cluster. The production from single ornamental products to practical products is a main route in flower industrial chain expansion and value appreciation. The main direction of flower industrial cluster to upgrade and form new specialized industry relies upon establishing flower production and processing bases for food, medicinal or industrial purposes and developing flower food products, drugs, health care products, cosmetics, spices, natural pigments, craft flower, etc.

\subsection{Production Normalization and Intensification}

Under condition of sophisticated demand and continuously rising costs of production factors, normalized planting and intensified management have become key factors for flower industrial cluster and enterprises to obtain 
continuous competition advantages. The production cycle of landscaping nursery is very long and it often takes five years or even ten years above to cultivate a nursery into a commodity. During this period, normalized planting, scientific trimming, fertilization and irrigation are key links to decide quality of products; for large-scale planting, reasonable configuration of factors and effective utilization of machines are key factors to reduce the cost. The basic measures of normalization and intensification include: (1) The local government, association and enterprises in industrial cluster location should make efforts to formulate prospective production and commodity standards for main cultivar of landscaping nursery and indicate direction for production and operation of cluster enterprise; (2) It is necessary to push the enterprises to implement intensified production and scientific precision management and help the enterprise to internalize the transformation from quantity expansion to quality promotion so as to adapt to the sophisticated market demand; (3) The measures of improving quality of factors and changing combination mode of factors shall be taken to achieve the goal of improving quality of products, reducing costs and enhancing benefits, in which, the main route to improve quality of factor includes strengthening of labor training, boosting quality of operation, organizing professional team, etc.

\subsection{Product Structural Adjustment and Quality Promotion}

The direction of market demand change is the direction of enterprise strategic adjustment. Therefore, the variety structural adjustment and quality promotion strategies shall focus on: (1) Based on the market characteristics that as different varieties may have large price fluctuation ranges in different years, partial of varieties may be supplied more than demand to enhance market uncertainty, then differentiation strategy shall be carried out among clusters and cluster enterprises to change homogenized and overcompetition situation and promote competitive power; to enhance anti-risk capability of enterprise, large-scale enterprise shall implement diversified planting strategies and small enterprises shall implement joint operation strategy. (2) As flower products are featured by long production period, so the decision of variety selection shall be prospective. Urbanization, ecologicalization, sophisticated resident income and other macro factors are vital decision factors. In the long run, the drought resistance, water saving and disease-resistant varieties with high stress resistance are most likely to become the main selections in future market. (3) To adapt to sophisticated and hypercritical demand, whether in medium and small enterprises or large-scaled enterprises, increasing the quality of product will be their strategic focus and long-term task.

\subsection{Innovative Incentives}

Innovative incentives are the core of cluster development strategies. No matter the promotion of product quality, production normalization and intensification, structural optimization or perfection of industrial system could not be separated from incentive of innovation. To implement innovation driven strategy, it is required to solve a series of problems, but the key point is to cultivate innovative capability of economic subject. Therefore, the strategic focus of innovation incentives includes: (1) To push combination of production, study and research is a key engine in agricultural cluster innovation. The majority of cluster knowledge innovation originates from market demand as well as independent research and development by large enterprise, however, the core enterprise could significantly improve its innovation capability through close combination of production, study and research [3]; (2) To promote cooperative innovation among clusters and enterprises: through adopting and cultivating core enterprise and promoting work division and cooperation between large enterprise and medium and small enterprises, it could effectively enhance online transfer and expansion of production knowledge and technical cluster of product and further improve innovation capabilities of medium and small enterprises or even cluster [9, 10]; (3) The government and enterprise need specialized investment in relation construction with scientific research institutions, independent developing capabilities and building of modern market system. Such kind of creation and expansion of new knowledge formed under specialized investment is the source to create cluster routine and maintain sustainable competitive advantage and vitality [11]; (4) The introduction of management and technical talents and increase of training strength could provide talent support and guarantee for flower enterprises in their promotion of management level and innovation capability.

\section{CONFLICT OF INTEREST}

The author confirms that this article content has no conflict of interest.

\section{ACKNOWLEDGEMENTS}

This work is supported by the National Social Science Fund Program (No. 13BJY111); Henan Provincial Philosophical and Social Science-Planned Program (No. 2012BJJ047); and Humanistic and Social Science Research Program of Henan Education Department (No.2014-ZD015).

\section{REFERENCES}

[1] W. M. Lee, and G. M. Schrock, "Rural Knowledge Clusters: The Challenge of Rural Economic Prosperity", State and Local Policy Program Staff Working Paper, 2002.

[2] F. Zheng, and C. Yu, "Entrepreneur and formation and evolution mechanism of Chinese rural industrial cluster," China Soft Science, no. 1, pp. 100-107, 2006

[3] E. Li, Y. Shi, and X. Li, "An analysis on agricultural innovative system structure on basis of agricultural industrial cluster-taking Yanling flower industrial cluster in Henan province as example," Economic Geography, vol. 32, no. 1, pp. 113-119, 2012.

[4] "Yanling modern brand-name and best-quality flower technological zone: Yanling fower industrial special report", 2011.

[5] G. Elisa, "Networks and Heterogeneous Performance of Cluster Firms," Chapter 8 in: K E Giuliani-Applied Evolutionary Economics and Economic Geography, 2007. 
[6] N. Cai, and J. Wu, "Online innovation capability and collective learning mechanism of industrial cluster," Research Management, vol. 26, no. 4, pp. 22-28, 2005.

[7] J. Zhu, "The process of transformation from farmer to enterprise under background of agricultural industrialization and its mechanism study," Kaifeng : Henan University, 2012.

[8] L. Wei, and J. Li, "Analysis for effects of social capital and human capital of agricultural industry cluster on farmers' income: based on the micro-data of tea industry cluster in anhui province," Issues in Agricultural Economy (Monthly), no. 12, pp. 41-47,
[9] N. Carbonara, "Innovation processes within geographical clusters: a cognitive approach," Technovation, vol. 24, no. 1, pp. 17-28, 2004

[10] L. Wei, and J. Li, "An analysis of the influence of cooperative relationship between participants in agricultural industry clusters on the income of the farmers and related businesses: taking anhui province as an example," Journal of Jiangxi University of Finance and Economics, no. 1, pp. 75-83, 2015.

[11] C. Miao, and Y. Wei, "Deepened work division, knowledge creation and industrial cluster growth," Geographical Research, vol. 24, no. 1, pp. 854-864, 2009.

Received: June 10, 2015

Revised: July 29, 2015

Accepted: August 15, 2015

(C) Wei Jianfeng; Licensee Bentham Open.

This is an open access article licensed under the terms of the (https://creativecommons.org/licenses/by/4.0/legalcode), which permits unrestricted, noncommercial use, distribution and reproduction in any medium, provided the work is properly cited. 